Reprod. Nutr. Dévelop., 1981, 21 (2), 289-292.

\title{
Etude de la synthèse des protéines totales et des protéines contractiles dans l'hypertrophie cardiaque expérimentale
}

\author{
par J. M. MOALIC, M. C. AUMONT, J. BERCOVICI, B. SWYNGHEDAUW \\ U 127 INSERM, Hôpital Lariboisière \\ 41, bd de la Chapelle, 75010 Paris.
}

Summary. Total and contractile protein synthesis during experimental cardiac hypertrophy.

The fractional turnover rate of the total protein extracted from cardiac rat ventricles has been measured by the continuous infusion flow technique in unanesthesized animals using ${ }^{3} \mathrm{H}$-lysine as a marker. This turnover rate approximated 12 p. 100 per day, doubled after aortic stenosis or aortic incompetence, and reached a peak within a week after stenosis and 2 weeks after incompetence. The fractional turnover rate of actin and myosin heavychains has been measured by the same technique using ${ }^{14} \mathrm{C}$-tyrosine as a marker and preparative gel electrophoresis to isolate the protein. This turnover rate approximated 22 and 9 p. 100 per day for myosin and actin, respectively, and doubled 4 days after aortic stenosis. It is suggested that a drop in contraction efficiency triggered protein synthesis in cardiac overloading.

L'augmentation de travail et de puissance qui est imposée au cœur par une surcharge de pression (ou systolique) ou de volume (ou diastolique) provoque un certain nombre de réactions d'adaptation dont la plus importante est l'hypertrophie dite compensatrice tant qu'elle compense l'augmentation de travail.

Bien que l'on ignore encore le mécanisme précis par lequel l'augmentation de travail stimule la croissance tissulaire, on sait déjà que c'est un mécanisme très rapide (Hatt, Ledoux, et Bonvalet, 1965) qui active à la fois l'Acide Ribo Nucléique (ARN)polymérase, la synthèse protéique, la captation et l'acylation des acides aminés, la synthèse des nucléotides ef celle des nucléosides (Zak et al., 1976).

On se propose ici d'étudier sur animal entier la synthèse protéique par la méthode de perfusion continue du précurseur radioactif qui est la seule méthode qui permette une mesure de vitesse de renouvellement sur un seul animal en évitant les phénomènes de recirculation de l'isotope. Deux modèles seront comparés, les sténoses de l'aorte ef les insuffisances aortiques.

\section{Matériel d'étude et technique.}

Les sténoses aortiques abdominales et les insuffisances aortiques sont réalisées chez des rats Wistar mâles de $200 \mathrm{~g}$, selon des techniques déjà publiées (Swynghe- 
dauw et al., 1980), Le groupe expérimental a été comparé à un groupe témoin apparié en fonction du poids ef ayant eu, soit une laparotomie, soit une ligature carotidienne. Le pourcentage d'hypertrophie est calculé pour les deux ventricules à partir d'une droite de régression poids du corps/poids des ventricules établie chez 172 témoins.

La technique de perfusion continue est celle de Garlick (Garlick ef al., 1975), réalisée chez le rat éveillé pendant $6 \mathrm{~h}$ avec un débit de $0,5 \mathrm{ml} / \mathrm{h}$.

Protocole 1 : La solution perfusée est du sérum physiologique contenant $10 \mu \mathrm{Ci}$ de lysine tritiée et $30 \mathrm{Ul}$ d'héparine par $\mathrm{ml}$. A la fin de la perfusion, le cour est rapidement prélevé, les ventricules sont broyés dans l'azote liquide, la poudre est portée à ébullition dans l'eau 5 min puis additionnée d'acide trichloracétique (TCA) à 10 p. 100. Plusieurs lavages éliminent les phospholipides, le collagène et l'ARN lié aux protéines. Le surnageant TCA et le culot final servent à déterminer après électrophorèse à haute tension à $\mathrm{pH} 1,81$ (Waterlow et Stephen, 1967 ; Swynghedauw ef al., 1980) la radioactivité spécifique de la lysine libre ou liée aux protéines.

Protocole 2 : la solution perfusée contient $42 \mu \mathrm{Ci} / \mathrm{ml}$ de tyrosine $\mathrm{C}^{14}$. A la fin de la perfusion le cœur est rapidement prélevé, et un tiers des ventricules subissent le même traitement que dans le protocole 1 afin de déterminer la radioactivité spécifique de la tyrosine libre ; le reste du tissu ventriculaire sert à préparer un culot de myofibrilles selon le protocole de Etlinger (Etlinger et al., 1975). Ces myofibrilles sont ensuite posées sur des plaques d'électrophorèse sur gel de polyacrylamide en présence de sodium-dodecyl-sulfate et mises à migrer selon la technique de Porzio et Pearson (1977) qui sépare la pluparł des protéines myofibrillaires. Après coloration et décoloration, les bandes correspondant aux chaînes lourdes de la myosine et à l'actine sont découpées et hydrolysées dans l'acide chlorhydrique $6 \mathrm{~N}$. La tyrosine est isolée à partir du surnageant TCA et à partir des hydrolysats, ce qui permet de mesurer la radioactivité spécifique libre et liée aux 2 protéines myofibrillaires de la tyrosine sur un fluorimètre à filtre (Garlick et al., 1975). Les 2 protéines isolées sont identifiées grâce à un étalon constitué par de l'actomyosine désensibilisée. Les comptages sont effectués après décoloration à l'eau oxygénée dans un liquide scintillant contenant $4 \mathrm{~g}$ de PPO ef $0,1 \mathrm{~g}$ de POPOP par litre de toluène.

La vitesse de renouvellement est calculée d'après Garlick et al. (1975) pour les protéines totales et pour les protéines myofibrillaires étudiées.

\section{Résultats.}

Le degré d'hypertrophie cardiaque après sténose aortique ou insuffisance aortique est très comparable, mais alors qu'on obtient un plateau à $24 \pm 4$ p. 100 après sténose en 8 jours, il faut 2 à 3 semaines pour atteindre $43 \pm 5$ p. 100 dans l'insuffisance aortique. La teneur en eau du tissu cardiaque reste inchangée.

La fraction des protéines du ventricule renouvelée par jour chez le rat normal est comprise entre $13,3 \pm 1,6 \mathrm{p}$. $100 \mathrm{chez}$ les rats de moins de $300 \mathrm{~g}$ et $12,3 \pm 1,7 \mathrm{p} .100$ chez les rats plus gros. Ce chiffre est plus bas que celui obtenu dans le cœur de rat avec de la tyrosine par Garlick et al. (1975), mais cet auteur n'effectue pas de lavages au TCA chaud du culot protéique. Il est plus élevé que celui rapporté chez le porc ou 
le chien (Garlick, Burk et Swick, 1976 ; Everett, Taylor ef Sparrow, 1977). Après surcharge cardiaque la radioactivité spécifique libre reste inchangée mais celle des protéines croît (tabl. 1). Le renouvellement des protéines est accéléré mais l'accélération, d'un facteur deux environ, est plus précoce, vers une semaine, après sténose qu'après insuffisance aortique (tabl. 1).

TABLEAU 1

Vifesse partielle de renouvellement des protéines cardiaques de rat (en p. 100 des protéines par jour)

\begin{tabular}{|c|c|c|c|c|c|c|c|c|}
\hline & \multirow[b]{2}{*}{ Témoins } & \multicolumn{3}{|c|}{ Sténoses aortiques } & \multicolumn{4}{|c|}{ Insuffisances aortiques } \\
\hline & & 1 sem. & $2 \mathrm{sem}$ & $\begin{array}{l}1-2 \\
\text { mois }\end{array}$ & 1 sem. & 2 sem. & 3 sem. & $\begin{array}{c}1-2 \\
\text { mois }\end{array}$ \\
\hline $\begin{array}{l}\text { Protëines totales } \ldots . \\
\text { Chaînes lourdes de la } \\
\text { myosine } \ldots \ldots \ldots \ldots \\
\text { Actine } \ldots \ldots \ldots \ldots \ldots \\
\text { Myosine/actine ....... }\end{array}$ & $\begin{array}{l}12,8 \pm 1,6 \\
22,9 \\
10,5 \\
2,18\end{array}$ & $\begin{array}{l}24,8 \pm 2 \\
40,0 \\
21,8 \\
1,83\end{array}$ & $22,2 \pm 3$ & $12,2 \pm 1$ & $17,0 \pm 2$ & $26,6 \pm 3$ & $28,5 \pm 4$ & $10,8 \pm 1$ \\
\hline
\end{tabular}

Le renouvellement des chaînes lourdes de la myosine est à peu près deux fois plus rapide (tabl. 1) que celui de l'actine dans le cœur de rat normal, ce qui est en accord avec les résultats trouvés par d'autres avec une technique d'injection unique. Quatre jours après une sténose aortique la vilesse de synthèse est doublée pour les deux protéines mais cette accélération semble porter plus sur l'actine que sur la myosine (résultats préliminaires) (tabl. 1).

\section{Discussion.}

Les résultats obtenus pour les protéines totales après sténose aortique sont en accord avec ceux publiés par de nombreux auteurs et en particulier par Evereft, Taylor et Sparrow (1977) qui utilisent la même méthode et trouvent après surcharge systolique du ventricule droit, une augmentation de la vitesse de renouvellement des protéines correspondantes dès les 24 premières heures. Il y a très peu de travaux qui concernent les surcharges diastoliques. Le seul travail est celui de Schreiber et al. (1975) qui, sur couur isolé travaillant in vitro, trouvent une accélération de la synthèse proféique une demi-heure après surcharge de pression alors qu'une surcharge de volume est sans effet.

Trois hypothèses peuvent être avancées pour expliquer le retard observé dans l'activation de la synthèse protéique dans les surcharges de volume : (i) la synthèse est déclenchée par une baisse du rendement ( $g$ de travail fourni par mole d'ATP hydrolysé) ; ce dernier est en effet optimum pour une vitesse de raccourcissement donnée et, pour des raisons de pure mécanique musculaire, une surcharge systolique va d'emblée diminuer vitesse et rendement alors qu'une surcharge diastolique va, dans un premier temps, accélérer la viłesse et améliorer le rendement (Sonnenblick, 1966 ; Goldspink, 1975) ; (ii) le mécanisme déclenchant est la surcharge de pression, cette dernière existant dans les surcharges de volume, mais pouvant mettre un certain 
temps à s'installer (Sokolow et Mac Ilroy, 1977) ; (iii) le mécanisme déclenchant est la contrainfe pariétale ( wall stress » = rayon $\times$ pression/épaisseur) laquelle augmente instantanément dans les surcharges de pression et au bout d'un certain temps dans les surcharges de volume. La différence très nette observée entre synthèse des chaînes lourdes de la myosine et actine est en accord avec celle observée par le groupe de Zak (Martin ef al., 1977) mais en désaccord avec d'autres publications (Funabiki ef Cassens, 1972). C'est en tous les cas la première fois que le renouvellement des protéines contractiles est mesuré par la méthode de perfusion continue. La légère différence observée, après sténose, entre les vitesses de renouvellement de l'actine et de la myosine peut être due à la multiplication des cellules non musculaires et à l'augmentation de la concentration en actine non musculaire qui pourrait s'ensuivre.

6e Réunion du groupe Développement I.N.R.A., Clermont-Ferrand/Theix, 22-23 mai 1980.

\section{Références}

ETLINGER J. D., ZAK R., FISCHMAN D. A., RABINOWITZ M., 1975. Isolation of newly synthesized myosin filaments from skeletal muscle homegenates and myofibrils. Nature, 255, 259-261.

EVERETT A. W., TAYLOR R. R., SPARROW M. P., 1977. Protein synthesis during right-ventricular hypertrophy after pulmonary-artery stenosis in the dog. Biochem. J., 166, 315-321.

FUNABIKI R., CASSENS R. G., 1972. Heterogenous turnover of myofibrillar protein. Nature, 236, 249.

GARLICK P. J., BURK T. L., SWICK R. W., 1976. Protein synthesis and RNA in tissues of the pig. Amer. J. Physiol., 230, 1108-1112.

GARLICK P. J., MILLWARD D. J., JAMES W. P. T., WATERLOW J. C., 1975. The effect of protein deprivation and starvation on the rate of protein synthesis in tissues of the rat. Bioch. biophys. Acta, 414, 71-84.

GOLDSPINK G., 1975. Biochemical energetics for fast and slow muscles, 173-185. In BOLIS L., MADDRELL H. P., SCHMIDT-NIELSEN K., Comparative physiology. Functional aspects of structural materials. North Holland Publ. Co, Amsterdam.

HATT P. Y., LEDOUX C., BONVALET J. P., 1965. Lyse et synthèse des protéines myocardiques au cours de l'insuffisance cardiaque expérimentale. Arch. Mal. Cour Vaisseaux, 58, 1703-1710.

MARTIN A. F., RABINOWITZ M., BLOUGH R., PRIOR G., ZAK R., 1977. Measurements of half-life of rat cardiac myosin heavy chain with leucyl-tRNA as precursor pool. J. biol. Chem., 252, 3422-3429.

PORZIO M. A., PEARSON A. M., 1977. Improved resolution of myofibrillar protein with SDS polyacrylamide gel electrophoresis. Bioch. biophys. Acta, 490, 27-34.

SCHREIBER S. S., ROTHSCHILD M. A., EVANS C., REFF F., ORATZ M., 1975. The effet of pressure or flow stress on right ventricular protein synthesis in the face of constant and restricted coronary perfusion. J. clin. Invest., 51, 1-11.

SOKOLOW M., Mac ILROY M. B., 1977. Clinical cardiology. Lange Medical, Los Altos Calif.

SONNENBLICK E. H., 1966. The mechanics of myocardial contraction, 173-250. In BRILLER S. A., CONN H. L. Jr., The myocardial cell. Univ. Penn. Press, Philadelphia.

SWYNGHEDAUW B., SCHWARTZ K., BERCOVICI J., BOUVERET P., LOMPRE A. M., THIEM N. V., LACOMBE G., 1980. Experimental systolic and diastolic overloading in rats : total protein turnover rate. Enzymatic and structural properties of myosin. Basic Res. Cordiol., 75, 143-148.

WATERLOW J. C., STEPHEN J. M. L., 1967. The measurement of total lysine turnover in the rat by intravenous infusion of $\mathrm{L}-\left(\mathrm{U}-{ }^{14} \mathrm{C}\right)$ lysine. Clin. Sci. 33, 489-506.

ZAK R., MARTIN A. F., REDDY M. K., RABINOWITZ M., 1976. Control of protein balance in hypertrophied cardiac muscle. Circul. Res., 38 (suppl. 1), 145-150. 